\title{
Новые разработки АО «ПКК Миландр" в области генераторов частоты
}

\author{
А. Цветков ${ }^{1}$
}

УДК 621.3.049 | ВАК 05.27.01

\begin{abstract}
С 2009 года в центре проектирования интегральных микросхем компании "Миландр" ведутся разработки микросхем активной части тактовых генераторов и синтезаторов на основе ФАПЧ. За эти годы было разработано и запущено в серию несколько микросхем активной части тактовых однократно-программируемых и термокомпенсированных генераторов серии 1316ГН. Также созданы и запущены в производство микросхемы синтезатора частоты до 12 ГГц 1508АС015 и синтезатора частоты со встроенным генератором, управляемым напряжением, до 6 ГГц 1508МТ015. В 2019 году компания "Миландр" выпустила на рынок серию микросхем 1316ММ активной части генератора с диапазоном выходных частот 25-860 МГц и миниатюрный тактовый радиационно-стойкий генератор ГК385-П с диапазоном выходных частот 10-60 МГц. Об этих новых разработках рассказывается в статье.
\end{abstract}



енератор ГК385-П (рис. 1) предназначен для применения в аппаратуре космического, специального и гражданского назначения для тактирования цифровых схем. Генератор работает в диапазоне температур от -60 до $85^{\circ} \mathrm{C}$, обладает высокой надежностью и повышенной стойкостью к специальным внешним воздействующим факторам (СВВФ). Устройство выпускается в стандартном 4-выводном металлокерамическом корпусе для поверхностного монтажа размером 7×5 мм. На данный момент освоено в производстве пятнадцать наиболее востребованных номиналов частоты в диапазоне от 10 до 60 МГц. По желанию заказчика может быть изготовлен генератор с любым номиналом частоты в указанном диапазоне.

В зависимости от исполнения генераторы могут формировать выходные сигналы различного типа: синус (SIN), КМОП, ТЛ. В устройствах предусмотрены два режима энергосбережения: "дежурный» или «простоя". В "дежурном" режиме управляющий вывод OE / SHDN отвечает за

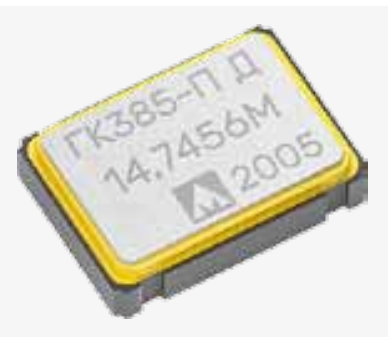

Рис. 1.

Внешний вид генератора ГК $385-\Pi$

АО «ПКК Миландр", ведущий инженер отдела разработки радиочастотных ИС и преобразователей, tsvetkov.alexey@ic-design.ru.
Таблица 1. Характеристики генератора ГК385-П

\begin{tabular}{|c|c|}
\hline Параметр & Значение \\
\hline $\begin{array}{l}\text { Диапазон номинальных } \\
\text { частот, МГц }\end{array}$ & От 10 до 60 \\
\hline Напряжение питания, В & $3,3 \pm 10 \%$ или $5,0 \pm 10 \%$ \\
\hline $\begin{array}{l}\text { Рабочий температурный } \\
\text { диапазон, }{ }^{\circ} \mathrm{C}\end{array}$ & От -60 до +85 \\
\hline Размер корпуса, мм & $7 \times 5$ \\
\hline $\begin{array}{l}\text { Максимальный ток } \\
\text { потребления, мА }\end{array}$ & $\begin{array}{l}35 \text { (при 3,63 В) } \\
\text { и } 55 \text { (при 5,5 В) }\end{array}$ \\
\hline Тип выходного сигнала & SIN, КМОП, ТТЛ \\
\hline $\begin{array}{l}\text { Точность настройки } \\
\text { номинальной частоты }\end{array}$ & $\begin{array}{l}\text { Не хуже } \pm 20 \text { ррm } \\
\text { (класс настройки 15) }\end{array}$ \\
\hline $\begin{array}{l}\text { Температурная нестабильность } \\
\text { рабочей частоты во всём } \\
\text { диапазоне рабочих температур }\end{array}$ & Не более $\pm 50 \mathrm{ppm}$ \\
\hline $\begin{array}{l}\text { Размах напряжения } \\
\text { в режиме SIN, B }\end{array}$ & $\begin{array}{l}\text { Не менее } 0,15 \\
\text { (при нагрузке } 50 \text { Ом) }\end{array}$ \\
\hline $\begin{array}{l}\text { Длительность фронта } \\
\text { в режимах КМОП, ТТЛ, нс }\end{array}$ & Не более 5 \\
\hline $\begin{array}{l}\text { Интегральный частотный } \\
\text { джиттер в полосе частот } \\
\text { от } 12 \text { кГц до } 20 \text { МГц, пс }\end{array}$ & $\begin{array}{l}\text { Не более } 2 \text { (типовое } \\
\text { значение), } \\
\text { не более } 8 \text { (макси- } \\
\text { мальное значение) }\end{array}$ \\
\hline
\end{tabular}




\section{Генератор ГК385-П-15ГР-3-А-10M-К}

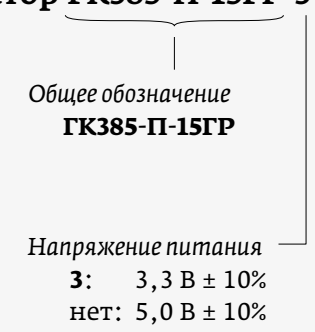

Приемка

K: "OTK"

нет: "ВП"

Выходная частота

10M: 10 МГц, допустимо от 10 до 60 МГц

- Тип выходного сигнала и управления

A: выход КМОП и управляющий сигнал ОЕ

Б: выход КМОП и управляющий сигнал SHDN

B: выход ТТЛ и управляющий сигнал $\mathrm{OE}$

г: выход ТТЛ и управляющий сигнал SHDN

д: выход SIN и управляющий сигнал OE

E: выход SIN и управляющий сигнал SHDN

генератора ГК385-П, которое необходимо указывать при заказе, представлено на рис. 2.

Одновременно с ГК385-П была выпущена серия бескорпусных микросхем 1316Мм, которые являются микросхемами активной части кварцевых генераторов с выходными частотами до 860 МГц. Серия состоит из шести микросхем. Их основные характеристики представлены в табл. 2.

Выбор режимов работы и типа выходного сигнала определяется комбинацией служебных площадок микросхемы.

В режиме КМОП можно только по-

Рис. 2. Обозначение генератора ГК385-П при заказе

работу только выходного драйвера и при подаче на этот вывод логической единицы на выход генератора подается выходной сигнал. В режиме «простоя» генератор полностью выключается при подаче на вывод OE/SHDN логического нуля. При подаче логической единицы генератор запускается и время выхода на рабочий режим составляет не более 10 мс. Основные характеристики генератора ГК385-П приведены в табл. 1. Обозначение делить частоту, для остальных типов выходного сигнала - только умножить.

Отличительной особенностью микросхем является блок умножителя частоты кварцевого генератора на 2 или на 4 (рис. 3). Для умножения частоты используются две внешние индуктивности, подключаемые к блоку через площадки. С помощью блока умножения из входной частоты (от 100 до 215 МГц) может быть сформирована



Рис. 3. Структурная схема блока умножителя 
выходная частота до 860 МГц. Также блок имеет режим прямого транслирования входной частоты на выход без умножения.

Блок состоит из двух каскадов умножения частоты на 2. При умножении на 4 происходит последовательное умножение на 2, затем еще раз на 2.

В зависимости от выбранного режима работы аналоговый мультиплексор выбирает точку съема синусоидального сигнала. Каждый из каскадов умножения частоты представляет собой перекрестно-связанный разбалансированный дифференциальный каскад (ПСРД), проходная
ВАХ которого имеет ярко выраженный максимум вблизи нулевого входного дифференциального напряжения. В результате, выходной ток ПСРД имеет явные пики при прохождении входного сигнала через 0. Поскольку за один период входной сигнал проходит через 0 дважды, спектр выходного тока ПСРД имеет хорошо выраженную составляющую второй гармоники входного сигнала. В качестве нагрузки для ПСРД выступает резонансный контур, образованный внешней индуктивностью и конденсаторами на кристалле. Резонансная частота контура настраивается на удвоенную частоту входного сигнала ПСРД.

Таблица 2. Параметры микросхем серии 1316MM

\begin{tabular}{|c|c|c|c|c|c|c|}
\hline Название параметра & 1316MM02 & 1316MM03 & 1316MM04 & 1316MM05 & 1316MM06 & 1316MM07 \\
\hline Тип выходного сигнала & $\begin{array}{l}\text { KMOП } \\
\text { LVDS } \\
\text { LVPECL } \\
\text { SIN }\end{array}$ & $\begin{array}{l}\text { КMOП } \\
\text { LVDS }\end{array}$ & $\begin{array}{c}\text { SIN } \\
\text { LVPECL }\end{array}$ & $\begin{array}{l}\text { КMOП } \\
\text { LVDS } \\
\text { LVPECL } \\
\text { SIN }\end{array}$ & $\begin{array}{l}\text { КMOП } \\
\text { LVDS }\end{array}$ & $\begin{array}{c}\text { SIN } \\
\text { LVPECL }\end{array}$ \\
\hline Выходная частота, МГц & $12,5-100$ & $50-860$ & $100-860$ & $0,78-100$ & $50-860$ & $100-860$ \\
\hline Тип схемы & \multicolumn{3}{|c|}{$\begin{array}{l}\text { Активная часть } \\
\text { генератора (ГУН) }\end{array}$} & \multicolumn{3}{|c|}{$\begin{array}{c}\text { Активная часть генератора } \\
\text { (тактовый) }\end{array}$} \\
\hline $\begin{array}{l}\text { Диапазон управляющего напряжения } \\
\text { (ГУН), в }\end{array}$ & \multicolumn{3}{|c|}{$1,65 \pm 1,65$} & \multicolumn{3}{|c|}{-} \\
\hline Перестройка частоты (ГУН) & \multicolumn{3}{|c|}{ Не менее $\pm 100 \times 10^{-6}$} & \multicolumn{3}{|c|}{-} \\
\hline Рабочая гармоника резонатора & \multicolumn{3}{|c|}{ 1-я гармоника } & \multicolumn{3}{|c|}{ 3-я гармоника } \\
\hline Частота гармоники кварца, МГц & $25-100$ & $100-215$ & $100-215$ & $25-100$ & $100-215$ & $100-215$ \\
\hline $\begin{array}{l}\text { Коэффициенты умножения } \\
\text { (блок умножения частоты) }\end{array}$ & - & $1,2,4$ & $1,2,4$ & - & $1,2,4$ & $1,2,4$ \\
\hline $\begin{array}{l}\text { Коэффициенты деления в режиме } \\
\text { КМОП }\end{array}$ & $1,1 / 2$ & $1,1 / 2$ & - & $\begin{array}{c}1,1 / 2 \\
1 / 4,1 / 8 \\
1 / 16,1 / 32\end{array}$ & $1,1 / 2$ & - \\
\hline Размер кристалла, мкм & \multicolumn{6}{|c|}{$1700 \times 1700$} \\
\hline Размер контактной площадки, мкм & \multicolumn{6}{|c|}{$80 \times 80$} \\
\hline Диапазон питающего напряжения, в & \multicolumn{6}{|c|}{$2,5 \pm 10 \% ; 3,0 \pm 10 \% ; 3,3 \pm 10 \%$} \\
\hline $\begin{array}{l}\text { Размах выходного напряжения } \\
\text { в режкиме SIN, мB }\end{array}$ & $\begin{array}{l}\text { Не менее } \\
\quad 800\end{array}$ & - & $\begin{array}{l}\text { Не менее } \\
\quad 800\end{array}$ & $\begin{array}{l}\text { Не менее } \\
\quad 800\end{array}$ & - & $\begin{array}{l}\text { Не менее } \\
800\end{array}$ \\
\hline $\begin{array}{l}\text { Коэффициент заполнения в режиме } \\
\text { КМОП, \% }\end{array}$ & $50 \pm 5$ & $50 \pm 5$ & - & $50 \pm 2$ & $50 \pm 5$ & - \\
\hline \multicolumn{7}{|c|}{ Динамический ток потребления, мА (не более): } \\
\hline в режиме SIN & 43 & - & 80 & 43 & - & 80 \\
\hline в режиме КмоП & 40 & 40 & - & 40 & 40 & - \\
\hline в режиме LVPECL & 80 & - & 80 & 80 & - & 80 \\
\hline в режиме LVDS & 80 & 80 & - & 80 & 80 & - \\
\hline
\end{tabular}




\section{m

\section{МИКРОСХЕМЫ ПРЕОБРАЗОВАТЕЛЕЙ}

Высокотехнологичная линейка от отечественного производителя

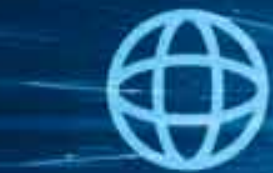

Индустриальное оборудование

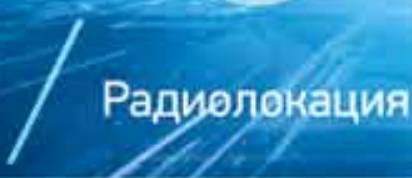

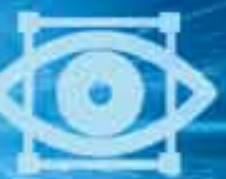

Системы

Системы
Системы связи



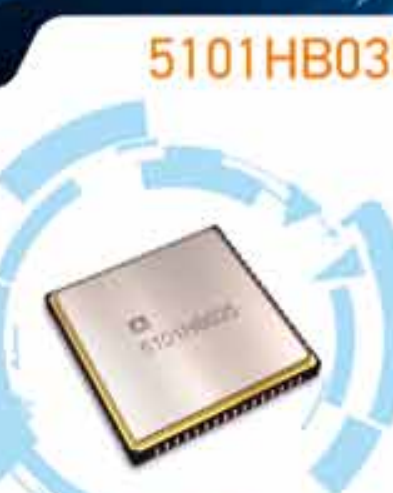

Высокоточная

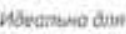

usiepumenued annopormypa



- 16 verterpanopos



- 8- rovolshix raranoe:

- B - single end канапов no напрніненис.

- 4- an $\phi$, нaranoo no нanpaimerono;

- Ulyana of $12 \mathrm{nHin}$ po $350 \mathrm{ntin}$

- Времн интегрированен от 10 мес до $1 \mathrm{c}$ :

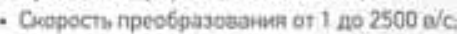

- Paspezanuar cnoco6hoctb 16/20 6in:

- Wум АLП 0.66 EMP (20 6nt) нa 50 sic npu

занорочсияеах дифференциалынеах оходах:

- Тон угеши по имесрательным оходам <10пA

- Напряжение питания $3,0-3,6$ B:

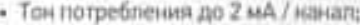

- Интерфейс SPI, DCH.

- Bстроенiны ИOH, RC-rehepatop.

- Temneparypuisí диariason müryc $60-85^{\circ} \mathrm{C}$
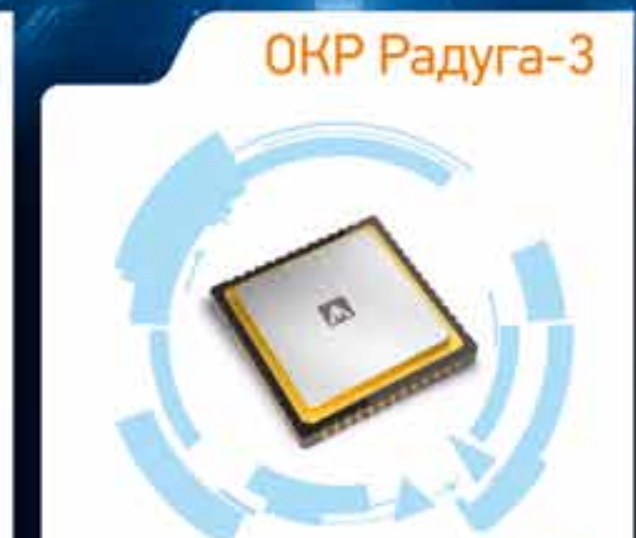

Высокоскоростная

- Разрядность 16 6ит

- Chopocta npeo6pasoianasar дo 80 Mes6/c:

- SNR 75 ja.

- SNR do 76.5 c aносшин onophim

4aกрнанением 1.25 B

- SFDR 89 A5:

- Без verтрливинга и автокалибровии:

- Совместияость < 1.8/2.5/3,3 В ПЛИС:

- Дenureni тантовoro curriana or 1 де 8;



источнеком питания $1,8 \mathrm{~B}$;

- Программируемыай встроенный источнй onopiaro напранения

- Температурный диапазон минус $60-85^{\circ} \mathrm{C}$
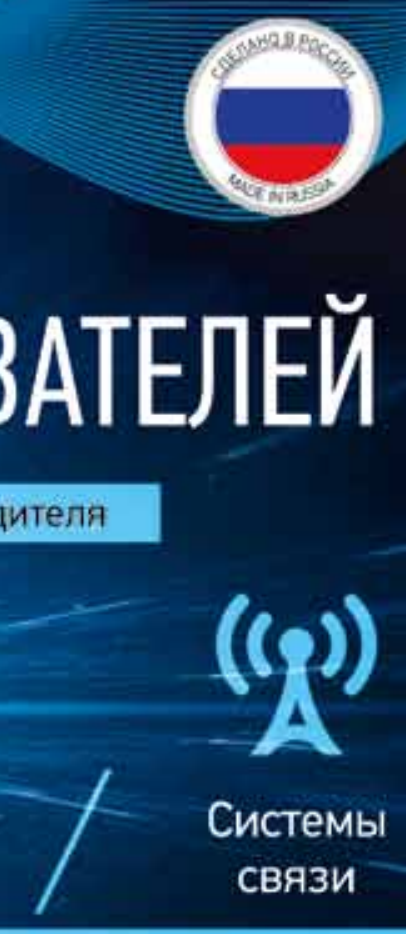

\section{(} rereTh



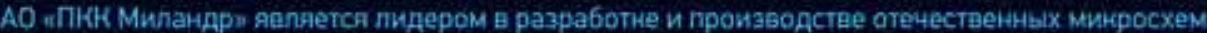
преобразователей. В нашем ассортименте представлены АЦП и ЦАП, соответствуюцие по своим техническим харантеристинам изделиям ведуцих мировых производителей. начинан от прецизионных многоразрядных сипма-депьта АЦП до высокоскоростных нонвейерных минросхем с частогами белее 125 МГц с повьшенными знсппуатационными характеристинами. 


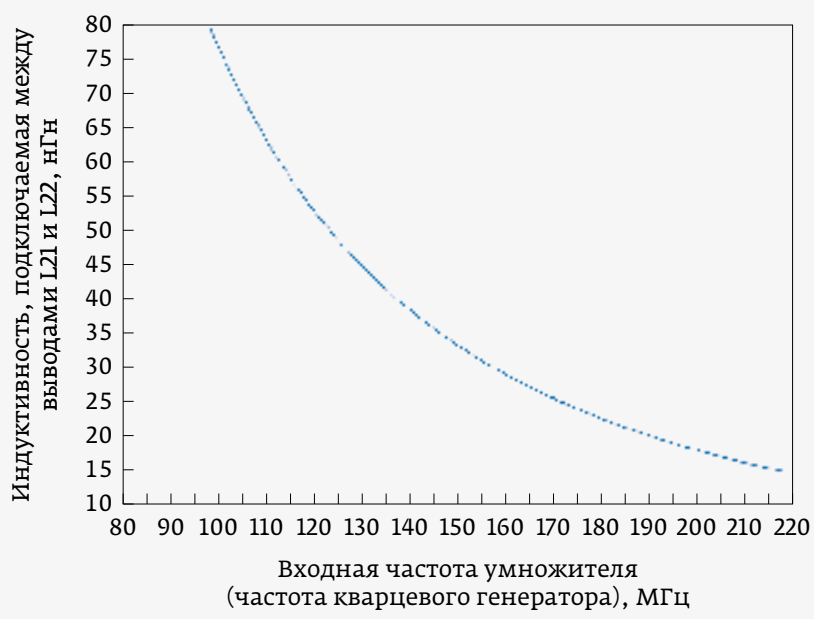

Рис. 4. Зависимость номинального значения индуктивности, подключаемой к выводам L21, L22, от входной частоты умножителя

Для компенсирования технологического разброса емкости конденсаторов в микросхеме, а также разбросов величин внешних индуктивностей L1, L2 в блок встроена система калибровки резонансной частоты (СКРЧ). Подстройка резонансной частоты осуществляется подключением необходимого числа конденсаторов в контур при помощи встроенных ключей и матрицы конденсаторов. Для заданной входной частоты

\section{Генератор ГК381-УН-7ДН2-80M-3,3С-А}

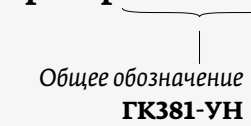

Точность настройки, $\times 10^{-6}$

5: \pm 10

6: \pm 15

7: \pm 20

8: \pm 30

Интервал температур, ${ }^{\circ} \mathrm{C}$

Ш: $-10 \ldots+85$

П: $-20 \ldots+70$

Б: $-30 \ldots+60$

B: $-40 \ldots+60$

C: $-40 \ldots+85$

Д: $-60 \ldots+85$

Нестабильность частоты, $\times 10^{-6}$

H: \pm 15

П: \pm 20

C: \pm 30

T: \pm 40

y: \pm 50
Упаковка

A: для автоматической сборки нет: для ручной сборки

Tuп выходного сигнала и управления

C: выход SIN

CM: выход КМОП

DS: выход LVDS

PE: ВЫХОД LVPECL

Напряжение питания

3,3: $3,3 \mathrm{~B} \pm 10 \%$

3,0: $3,0 \mathrm{~B} \pm 10 \%$

2,5: $2,5 \mathrm{~B} \pm 10 \%$

Выходная частота

80M: 80 МГц

- Предел перестройки, $\times 10^{-6}$

1: \pm 80

2: \pm 90

3: \pm 100
Рис. 6. Обозначение генератора ГК381-УН при заказе

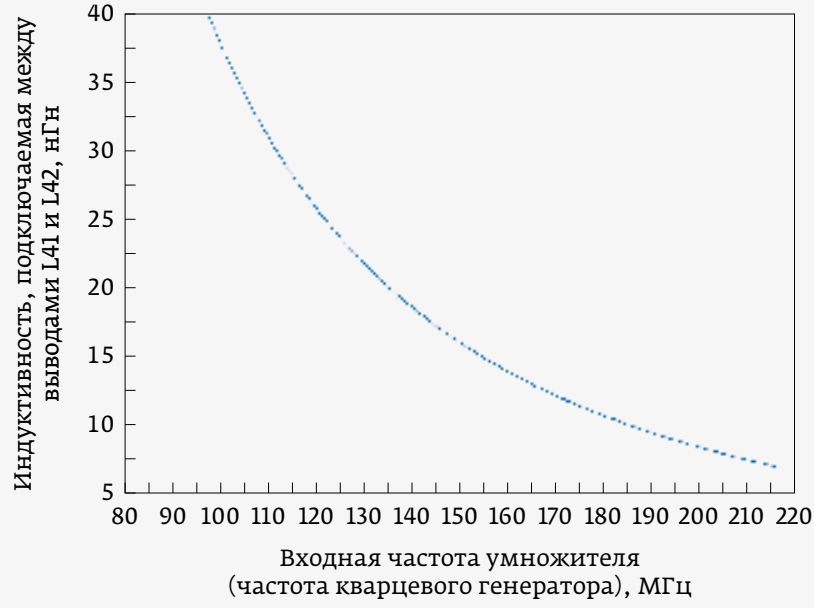

Рис. 5. Зависимость номинального значения индуктивности, подключаемой к выводам L41, L42, от входной частоты умножителя

и установленной индуктивности СКРЧ выбирает необходимый код управления матрицей конденсаторов по критерию максимальной амплитуды на резонансном контуре. Система калибровки запускается при каждом включении микросхемы. Для детектирования амплитуды на резонансном контуре используется встроенный детектор амплитуды. По окончании работы СКРЧ в регистры хранения записываются выбранные коды управления матрицами конденсаторов и выдается флаг готовности, по которому частота поступает на выход микросхемы.

При работе в режиме умножения на 4 необходима установка обеих индуктивностей L1 и L2. При работе в режиме умножения на 2 достаточно установки индуктивности L1. При работе в режиме транслирования частоты установка индуктивностей не обязательна. Номинальные значения индуктивностей в зависимости от входной частоты умножителя представлены на рис. 4 и 5.

Микросхемы серии 1316мМ уже применяются АО "Завод "Метеор" в производстве современных генераторов ГК380-П и ГК381-УН для поверхностного монтажа размером 7×5 мм (обозначение генератора ГКз81-УН, которое необходимо указывать при заказе, представлено на рис. 6). 


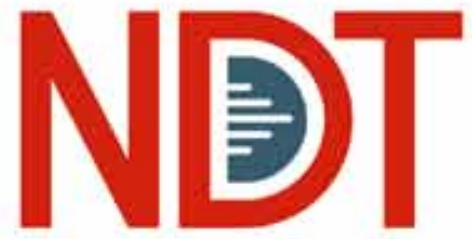

RUSSIA

NDT Russia

20-я Международная выставка оборудования

для неразрушающего контроля

27-29

октября

2020

Москва,

Крокус Экспо


Oprativaarop - komnanens MVK Obuc is Cankt-Merepbypre

NV

+7 (812) $3806010 / 00$ ndt@imvk.ru
Запросите

условия участия:

ndt-russia.ru 